\title{
Increased synthesis of indoleamine-2,3-dioxygenase protein is positively associated with impaired survival in patients with serous-type, but not with other types of, ovarian cancer
}

\author{
MIHO TAKAO $^{1}$, AIKOU OKAMOTO ${ }^{1,2}$, TAKASHI NIKAIDO ${ }^{3}$, MITSUYOSHI URASHIMA ${ }^{4}$, \\ SATOSHI TAKAKURA ${ }^{1}$, MISATO SAITO ${ }^{1}$, MOTOAKI SAITO ${ }^{1}$, SANSHIRO OKAMOTO $^{1}$, \\ OSAMU TAKIKAWA ${ }^{6}$, HIROSHI SASAKI ${ }^{1}$, MAKOTO YASUDA ${ }^{1}$, \\ KAZUNORI OCHIAI $^{1,5}$ and TADAO TANAKA ${ }^{1}$
}

\begin{abstract}
Departments of ${ }^{1}$ Obstetrics and Gynecology, ${ }^{2}$ Gene Therapy, Institute of DNA Medicine, ${ }^{3}$ Pathology, ${ }^{4}$ Division of Clinical Research and Development and ${ }^{5}$ Department of Clinical Oncology, The Jikei University School of Medicine, Minato-ku, Tokyo; ${ }^{6}$ National Institute for Longevity Sciences, National Center for Geriatrics and Gerontology, Aichi, Japan
\end{abstract}

Received December 19, 2006; Accepted March 2, 2007

\begin{abstract}
We previously reported that indoleamine-2,3dioxygenase (IDO) is associated with paclitaxel resistance and that IDO serves as a marker of poor prognosis in ovarian serous adenocarcinomas (SA). In this study, to explore the role of IDO in the development of various histological types of ovarian cancer, we further examined IDO expression not only in SA but also in other types of ovarian cancers. Expression of IDO protein was analyzed by immunohistochemistry for a total of 122 ovarian cancers including 40 SA, 67 clear cell adenocarcinomas (CCA), and 15 endometrioid adenocarcinomas (EA) with informed consent. Among these cases, there were $11 \mathrm{CCA}$ accompanied with endometriosis and 60 cases with lymph node metastasis. We classified the samples into four categories by IDO staining pattern. IDO staining was positive in $57.5 \%$ of SA, $49.2 \%$ of CCA, and $73.3 \%$ of EA, respectively. The Kaplan-Meier survival curve showed a clear relationship between staining score and overall survival for patients with advanced (stages III and IV) SA $(n=33)$ who underwent optimal surgery and paclitaxelcarboplatin (TC) chemotherapy as a first-line regimen. There was no association between IDO staining score and overall survival in the CCA cases. Eight of 11 cases $(72.7 \%)$ of CCA accompanied by endometriosis presented identical staining patterns of IDO between CCA and endometriosis. In 43 of 60 cases $(71.6 \%)$ with lymph node metastasis, the staining
\end{abstract}

Correspondence to: Dr Aikou Okamoto, Departments of Obstetrics and Gynecology, and Gene Therapy, Institute of DNA Medicine, The Jikei University School of Medicine, Nishishimbashi 3-25-8, Minato-ku, Tokyo 105-8461, Japan

E-mail: aikou@jikei.ac.jp

Key words: indoleamine-2,3-dioxygenase, ovarian cancer, immunohistochemistry, serous adenocarcinoma, paclitaxel, endometriosis patterns of IDO showed a correspondence between the primary lesion and metastatic site. These results suggested that the increased synthesis of IDO protein was positively associated with impaired survival only in the serous type of ovarian cancer.

\section{Introduction}

Ovarian cancer is one of the leading causes of death related to gynecologic malignancies. Despite various treatment advances, most patients will relapse after achieving complete clinical response, and the disease is incurable in the majority of these patients (1). Therefore, countering drug resistance is essential for ovarian cancer management.

We previously reported that IDO expression screened with the GeneChip system was positively associated with chemoresistance and impaired survival in patients with advanced SA (2). IDO is an enzyme that degrades the essential amino acid tryptophan. Uyttenhove et al reported that most human tumors including prostate, colorectal, pancreatic and cervical carcinomas constitutively express IDO, and that expression of IDO by immunogenic mouse tumor cells prevents their rejection by preimmunized mice. This effect was accompanied by a lack of accumulation of specific $\mathrm{T}$ cells at the tumor site and could be partly reverted by systemic treatment of mice with an inhibitor of IDO, in the absence of noticeable toxicity. They suggested that the efficacy of therapeutic vaccinations of cancer patients might be improved by concomitant administration of an IDO inhibitor (3). Muller et al reported that IDO inhibition acted cooperatively with diverse chemotherapeutic agents to effectively promote the regression of established breast tumors that were refractory to chemotherapy (4). They used MMTV-Neu mice, a wellestablished transgenic mouse model of breast cancer, and showed that combining the IDO inhibitor 1-methyl-DLtryptophan with paclitaxel resulted in a significant decrease of tumors compared with the administration of paclitaxel alone. In addition, they showed that 1-methyl-DL-tryptophan 
with cisplatin also resulted in a significant decrease in tumors compared with cisplatin alone.

In this study, to explore the role of IDO in the development of various histological types of ovarian carcinogenesis, we examined IDO expression not only in SA but also in other types of ovarian cancers and investigated the correlations between IDO expression patterns and clinicopathological features. Moreover, we further examined IDO expression in metastatic lesions and in endometriotic lesions.

\section{Materials and methods}

Cases and surgical samples. The Jikei University School of Medicine Ethics Review Committee approved the study protocol, and informed consent was obtained from all patients. A total of 122 ovarian cancer surgical specimens were obtained at The Jikei University Hospital and The Jikei Kashiwa Hospital. Ovarian cancer patients treated by primary laparotomy between 1988 and 2005 were identified from case records of the Department of Obstetrics and Gynecology at The Jikei University School of Medicine. Tumors were histologically classified according to the World Health Organization international system and staged according to the International Federation of Gynecology and Obstetrics.

The tumors included $40 \mathrm{SA}$ (32.8\%), 67 CCA (54.9\%), and 15 EA $(12.3 \%)$. The $40 \mathrm{SA}$ did not include 24 cases which were analyzed in our previous report (2). Among the 67 CCA, there were 11 cases accompanied by endometriosis. None of the 122 cases included borderline malignancy. There were 52 stage I cases (42.6\%), 7 stage II cases (5.7\%), 54 stage III cases $(44.3 \%)$ and 9 stage IV cases $(7.4 \%)$. There were 60 cases with metastasis to other organs.

Immunohistochemistry. For the immunohistochemical study, formalin-fixed paraffin-embedded sections were used. The immunohistochemical reactions were performed using Ventana Benchmark $^{\mathrm{TM}}$ autostainer (Ventana Medical Systems, Inc., Tuscon, AZ, USA) and visualized with Ventana DAB Universal Kit with diaminobenzidine as the chromogen. A monoclonal antibody against IDO (1:1000) was used. The antigen retrieval procedure was performed in a microwave oven in Dako antigen retrieval solution (Dako Cytomation, Glostrup, Denmark) for $10 \mathrm{~min}$ at $95^{\circ} \mathrm{C}$ in order to obtain efficient staining. The sections were developed with 3,3'diamino-benzidine with $0.3 \% \mathrm{H}_{2} \mathrm{O}_{2}$ and counterstained with hematoxylin. Positive and negative controls were tested in parallel for each staining. We classified the samples into four categories by IDO staining pattern including negative, sporadic, focal, and diffuse. We classified the samples as sporadic when positive stained cells scattered sporadically in the tumor lesion, and the incidence of positive cells in total cells corresponded to a low percent per slide. Similarly, samples were classified as focal when positive stained cells localized in several particular sites in the tumor lesion, and the incidence of positive cells in total cells corresponded to 20-30\% per slide. Diffuse samples were characterized by positive stained cells spread over a wide area in the tumor lesion, and by the incidence of positive cells corresponding to $>50 \%$ per slide. We also established a scoring system which considered both staining pattern and intensity: negative, 0; sporadic, 1-3; focal, 4-6, and diffuse, 7. M.T., A.O. and a pathologist T.N. read the slides independently.

Survival analysis. The survival curves of the patients were compared using the Kaplan-Meier method and analyzed by the log-rank test using Stata 9.0 (Stata Corporation, College Station, TX). The time of overall survival (OS) was defined from the date of primary laparotomy to the date of patient death or last follow-up. The relationship between the staining pattern of IDO and the clinicopathological features was determined using the $\chi^{2}$ test. $p<0.05$ was accepted as statistically significant.

\section{Results}

Immunohistochemistry. Positive IDO expression including sporadic, focal, and diffuse patterns was detected in 23 of 40 (57.5\%) SA, 33 of 67 (49.2\%) CCA, and 11 EA (73.3\%). Positive staining of tumor cells was much more prominent than that of non-cancerous cells in all sporadic, focal, and diffuse patterns. Regarding clinical stages, positive IDO expression was detected in 28 stage I cases (53.8\%), 4 stage II cases $(57.1 \%), 30$ stage III cases $(55.5 \%)$ and 4 stage IV cases $(44.4 \%)$. IDO expression was detected in 4 of 6 stage I, 1 of 1 stage II, 15 of 28 stage III and 2 of 5 stage IV cases in SA, and it was detected in 17 of 38 stage I, 3 of 6 stage II, 11 of 19 stage III and 2 of 4 stage IV cases in CCA.

Cases of SA. IDO expression was further confirmed using surgical samples obtained from 33 patients with stage III or IV SA treated with a TC regimen. The size of the residual tumors at first surgery was $<2 \mathrm{~cm}$ in all 33 cases. There were 16 negative, 9 sporadic, 7 focal cases, and 1 diffuse case (Fig. 1).

OS was compared between patients with stage III disease $(n=28)$ and stage IV disease $(n=5)$, and no significant difference was noted. Next, Kaplan-Meier survival curves were generated based on the IDO staining pattern. Staining patterns of IDO were related to impaired survival (log-rank test, $\mathrm{p}=0.0191$ ) (Fig. 2). The $50 \%$ survival time of patients classified as negative, sporadic, focal, and diffuse was 33, 29, 17, and 11 months, respectively. Moreover, statistical analysis showed significant differences among all of the scores (data not shown).

Cases of EA. In a similar way, IDO expression was analyzed using surgical samples obtained from patients with stage III or IV ovarian EA undergoing a TC regimen. The KaplanMeier survival curves showed no significant relationship with IDO expression (data not shown).

Cases of CCA. Firstly, IDO expression was examined using surgical samples obtained from 34 patients with stage III or IV CCA treated with cisplatin and CPT-11 (P-CPT regimen). Kaplan-Meier analysis showed no significant difference between IDO staining pattern and OS in CCA with P-CPT treatment. Next, surgical samples obtained from 8 patients with stage III or IV CCA treated with a TC regimen were examined for IDO expression. Kaplan-Meier analysis showed no significant difference between IDO staining 
A

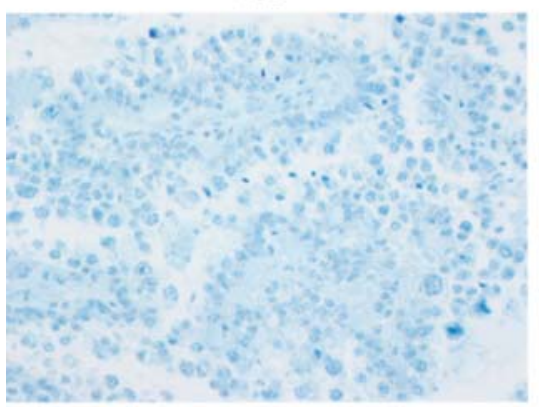

C

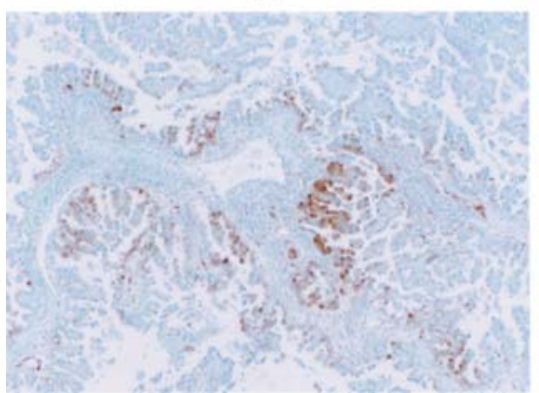

B

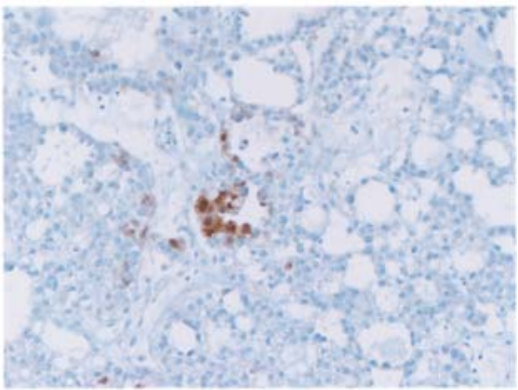

D

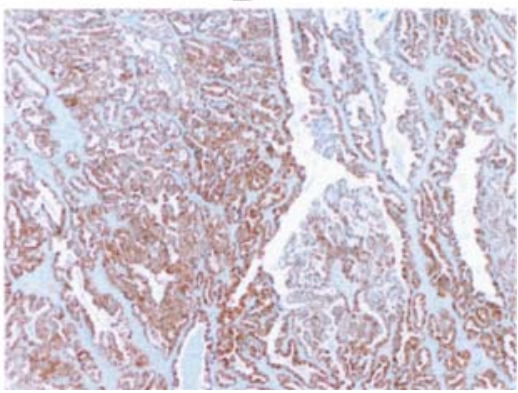

Figure 1. IDO protein expression in ovarian cancer lesions as detected by immunohistochemical staining. The staining patterns were classified as negative (A), sporadic (B), focal (C) or diffuse (D).

Log-rank test: $\mathrm{p}=0.0191$

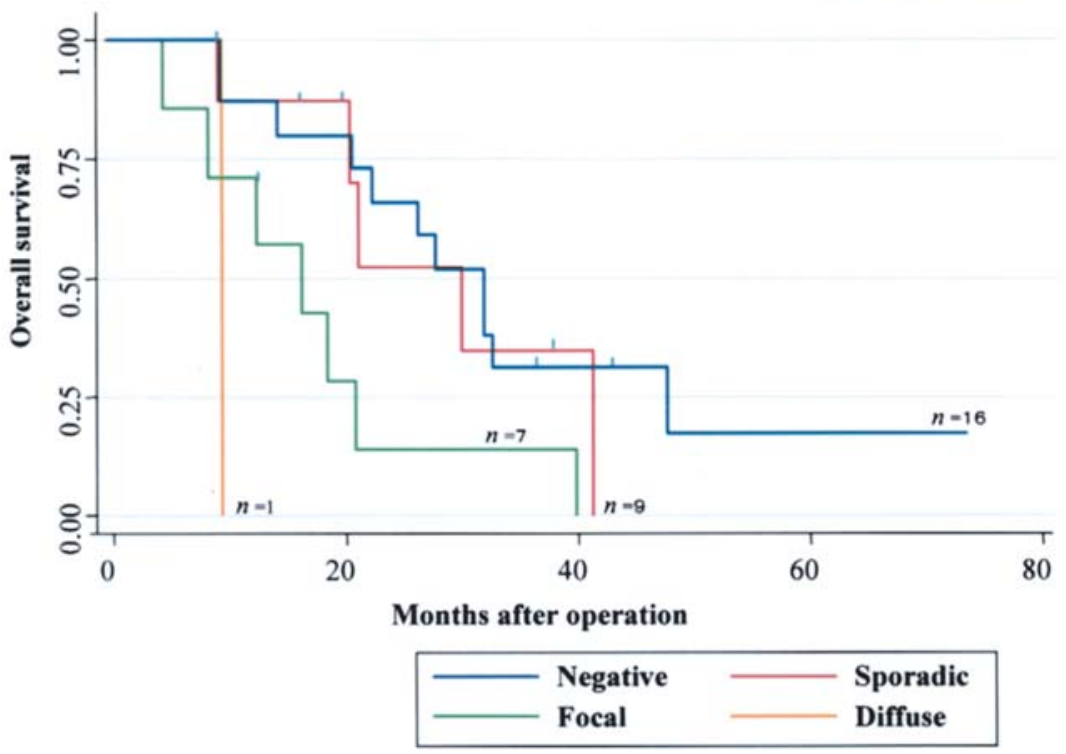

Figure 2. Patterns of IDO expression in serous adenocarcinoma and overall survival using the Kaplan-Meier method. The choice of patient samples was restricted to cancer stages III and IV. Staining patterns of IDO were related to impaired survival (log-rank test, $\mathrm{p}=0.0191)$. The $50 \%$ survival time of patients classified as negative, sporadic, focal, and diffuse was 33, 29, 17, and 11 months, respectively.

pattern and OS in CCA with a TC regimen. There have been many reports indicating a relationship between CCA and endometriosis. In this study, there was an obvious coexistence of CCA with endometriosis in 11 of 67 cases, and IDO expression in the cancerous lesion was compared with that in the endometriotic lesion. The staining patterns of IDO in the former and the latter corresponded to each other in 8 of 11 cases $(72.7 \%)$. In addition, 7 of 8 cases with such correspondence showed negative staining, and the remaining one of the 8 cases showed positive IDO staining in both the cancerous and endometriotic lesions. On the other hand, 3 of 11 cases did not display the same staining pattern between both lesions (Fig. 3). In the cases of CCA with endometriosis, we investigated whether age, FIGO stage, tumor size, serum CA125 or CA19-9 level, family history, smoking or drinking habits, history of pregnancy, or history of 


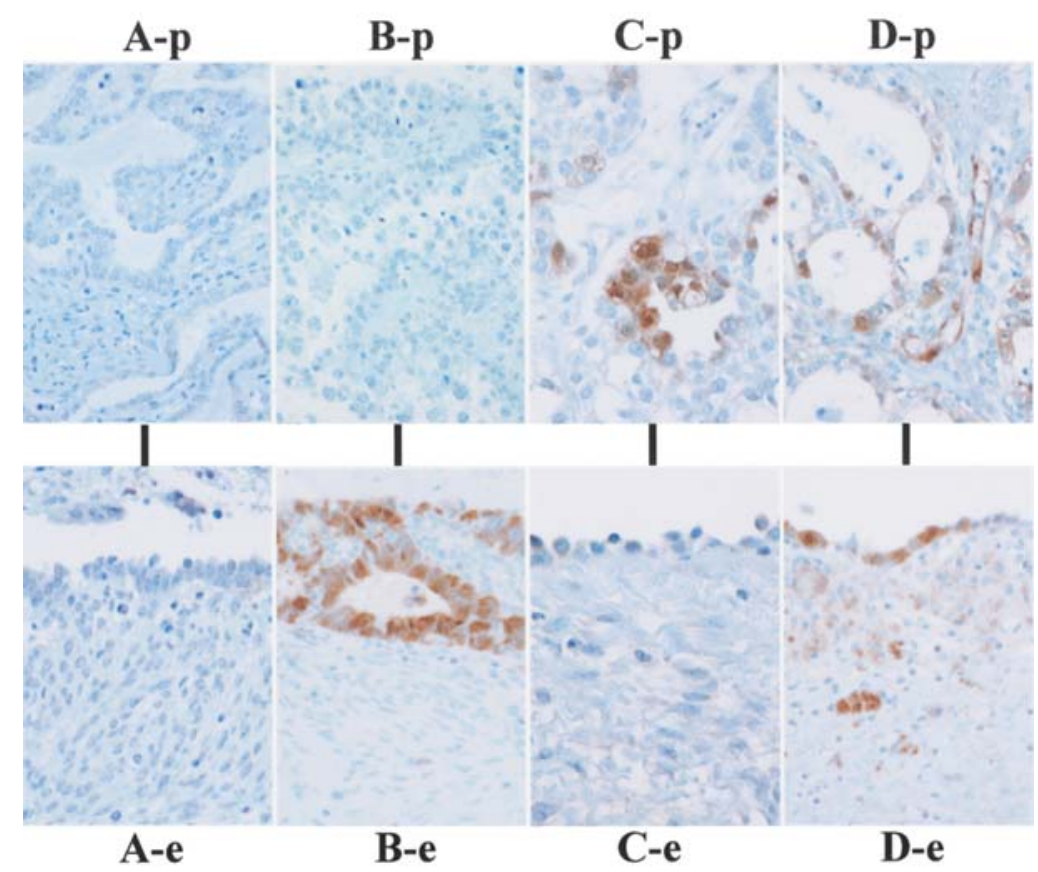

Figure 3. IDO protein expression in clear cell adenocarcinoma (CCA) lesions (upper) and endometriotic lesions (lower). The staining pattern of IDO in the CCA lesion corresponded to that in the endometriotic lesion in 8 of 11 cases (72.7\%) of CCA accompanied by endometriosis. p, primary lesion; e, endometriotic lesion.

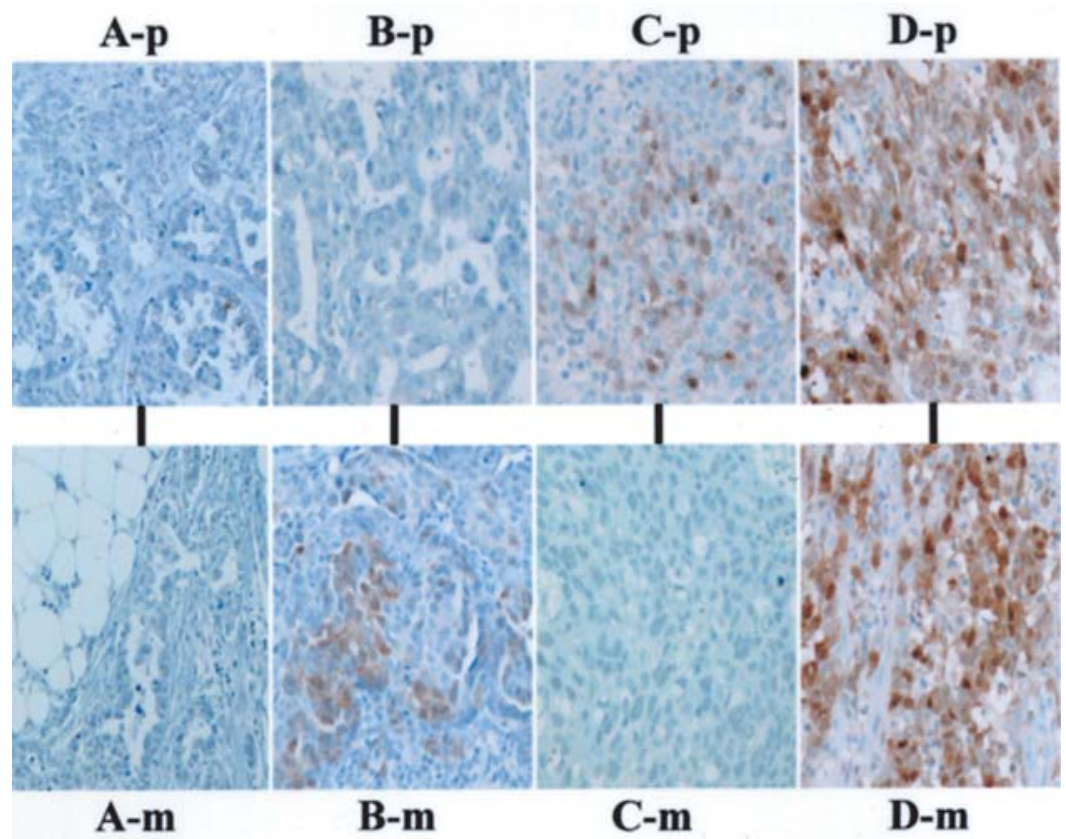

Figure 4. IDO protein expression in primary lesions (upper) and metastatic sites (lower). The histological types include clear cell adenocarcinoma (CCA) (A), serous adenocarcinoma (B), endometrioid adenocarcinoma (C), and serous adenocarcinoma (D). p, primary lesion; m, metastatic sites.

treatment of endometriosis were related to the correspondence with the staining pattern. There was no association between the cases regardless of the correspondence to the staining patterns.

Comparison of the primary lesion and metastatic site. Among 122 cases of all histological types, 60 had metastasis to other organs (e.g., the uterus, lymph node, omentum, small intestine, skin, and liver). We compared the staining pattern of IDO in the primary lesion with the staining pattern of IDO in the metastatic site in 43 of 60 cases (71.6\%) (Fig. 4). Sixteen of the 43 cases showed a positive staining pattern of IDO both in the primary lesion and in the metastatic site. Twenty-seven of the 43 cases showed a negative staining pattern of IDO in both lesions. Ten of 60 cases stained negative for IDO expression in the primary lesion and 
Table I. IDO expression in primary lesions and metastatic sites.

\begin{tabular}{|c|c|c|c|c|}
\hline Lesion & \multicolumn{4}{|c|}{ IDO expression } \\
\hline Primary & Negative & Negative & Positive & Positive \\
\hline Metastatic & Negative & Positive & Negative & Positive \\
\hline Number of cases & 27 & 10 & 7 & 16 \\
\hline
\end{tabular}

positive in the metastatic site. Seven of 60 cases displayed positive IDO expression in the primary lesion and negative in the metastatic site (Table I). Staining patterns of these 60 cases with metastasis to other organs had no correlation with age, histology, histological grade, FIGO stage, tumor size, serum CA125 and CA19-9 levels, family history, smoking or drinking habits, or history of pregnancy.

\section{Discussion}

In this study, we confirmed that IDO expression was positively associated with impaired survival only in SA while it was not associated with other types of ovarian cancer. For this reason, IDO was originally extracted from surgical samples of SA in our previous study (2). In general, gene alterations are related to histological subtypes. For example, we reported that 116 of 9121 genes showed significantly different expression by cDNA microarray analysis between SA and mucinous adenocarcinoma (MA) (5). In addition, $K$-RAS mutations occured more frequently in MA than in non-MA such as SA (6-10). Dent et al reported that the pattern of genomic alterations found by comparative genomic hybridization in CCA was distinct from that seen in other ovarian histological subtypes (11). Collectively, these reports suggest that gene alterations are related to the histological subtypes in ovarian cancer. In this study, the incidence of IDOpositive expression differed among the histological subtypes.

The incidence of the focal-diffuse patterns of IDO staining was $24.2 \%$ in stage III-IV SA, which is similar to the incidence of chemo-resistance in SA with a paclitaxel-based regimen (12). Moreover, IDO expression was positively associated with impaired survival in SA. Therefore, we believe that IDO is a good prognostic marker for SA.

CCA has clinically distinct features compared to other histological types, including SA. It tends to be associated with a poorer response rate to chemotherapy and with a poorer prognosis. The identification of CCA histology has been shown to be an indicator of poor prognosis in many studies $(11,13,14)$, with an inferior response to platinum-based chemotherapy (15). As previously mentioned, the pattern of genomic alterations found in CCA is distinct from that seen in other ovarian histological subtypes. Schwartz et al reported a detailed study showing that 73 genes were 2- to 29-fold overexpressed in CCA compared with the other major histological types of ovarian carcinoma (16). We examined IDO expression in surgical samples obtained from patients with stage III or IV CCA undergoing a P-CPT regimen. In our study of IDO expression, there was no significant association between IDO staining pattern and OS in CCA with a P-CPT or TC regimen. This result means that IDO expression may not be a prognostic marker for CCA, in contrast to SA.

Regarding prognostic markers for CCA, Tsuda et al reported that the $A B C F 2$ gene and its product is a potential prognostic marker for CCA and its expression is correlated with chemo-response in patients with CCA (17). In a previous study, we identified 8 genes that showed reproducible overexpression in SA, as indicated by real-time RT-PCR among GeneChip-selected genes (2). These 8 genes did not include $A B C F 2$ or $A B C F 2$-related genes. These results suggested that chemo-response factors are related to the histological subtype in ovarian cancer.

In this study, the staining pattern of IDO in the cancerous lesion of CCA corresponded to that in the accompanying endometriotic lesion in $>70 \%$ of the cases. There have been many reports indicating that there is a relationship between CCA and endometriosis (18). The development of endometriosis due to the menstrual dissemination of endometrial tissue into the peritoneal cavity is the theory most widely accepted to explain this as a kind of transplantation. IDO is an important enzyme in the regulation of immune responses, and cells which express IDO can suppress T cell proliferation and promote immune-tolerance by depleting tryptophan locally (3). IDO expression in endometriotic lesions plays a role as a suppressor of the immune system locally, resulting in the dissemination of endometriosis. In addition, endometriosis is considered to be a possible precancerous disease. Sekigawa et al reported that $K-R A S$ mutations were detected in CCA, but not in normal or atypical endometrial tissue (19). Very recently, Kato et al reported that HNF-1ß is an excellent molecular marker for ovarian clear cell tumors, including benign, borderline and malignant lesions (20). In this study, 8 of 11 cases of CCA accompanied by endometriosis presented identical staining patterns of IDO in CCA and endometriosis, suggesting that endometriosis is a precursor of CCA. Further studies should focus on the role of the IDO gene in the dissemination of endometriosis.

The staining pattern of IDO in the primary lesion corresponded to that in the metastatic site with an incidence of $71.6 \%$. Munn et al reported that human antigen-presenting cells that express IDO inhibit $\mathrm{T}$ cell proliferation (21). IDO expression by tumor cells or by tumor-associated antigenpresenting cells might inhibit $\mathrm{T}$ cell responses to tumor antigens by suppressing $\mathrm{T}$ cell priming in tumor-draining lymph nodes, or by rendering activated effector $\mathrm{T}$ cells ineffective at the site of tumor growth $(3,4,22,23)$. In our study, there were some cases where IDO expression was positive in the primary lesion and negative in the metastatic 
site. We speculate that unknown genes played important roles in the process of metastasis in these cases. There have been several reports on genes associated with the metastatic process in ovarian cancer, and candidate genes include bikunin, nm23, c-erbB2, and p53-associated genes (24-26). Further studies should focus on exploring the associations of these genes, including the IDO gene, in primary tumors and corresponding metastatic tumors.

According to the results obtained in this study, we hypothesize that IDO may play important roles in the development and the therapeutic susceptibility of ovarian cancer. Firstly, IDO plays a role as a suppressor of $\mathrm{T}$ cells, and secondly, it plays a role in chemo-resistance. Muller et al reported that IDO inhibition acted cooperatively with diverse chemotherapeutic agents to effectively promote the regression of established breast tumors that were refractory to chemotherapy. They also showed that loss of the BAR adapterencoding gene Binl elevated the signal transducers and activators of transcription STAT1 and nuclear factor- $\mathrm{B}$ dependent expression of IDO (4). Further studies should focus on searching for key molecules acting together with IDO in the possible effects on chemo-resistance, including nuclear factor- $\mathrm{kB}$ and $\mathrm{Akt} 2$.

In recent years there have been numerous reports concerning various biomarkers for ovarian cancer based on advances in genomic and proteomic technology. The material used to detect the biomarkers has included peripheral blood, plasma, and serum. Ovarian carcinoma has the worst prognosis among gynecological malignancies because most cases are not diagnosed until an advanced stage. Hence, the discovery of biomarkers for early-stage ovarian cancer is a very important issue. Luo et al reported that high expression of human kallikrein 10 in serum was strongly associated with SA $(27,28)$. Takano et al reported that the cytoplasmic overexpression of MUC1 might be an indicator of resistance to platinum-based chemotherapy and a prognostic marker in ovarian cancer (29). Matsuzaki et al reported that low preoperative plasma bikunin was strongly associated with a poor response to chemotherapy in ovarian cancer (30). This study demonstrated that IDO expression was positively associated with impaired survival in patients with SA, but not other types of ovarian adenocarcinoma. IDO thus may be useful as a new biomarker of SA.

\section{Acknowledgements}

This study was supported in part by the Japanese Ministry of Education, Science, Sports, and Culture; and the Japanese Ministry of Health, Labour, and Welfare. We thank Ms Misako Shirai for the technical help with the immunohistochemical staining.

\section{References}

1. Lister-Sharp D, McDonagh MS, Khan KS and Kleijnen J: A rapid and systematic review of the effectiveness and costeffectiveness of the taxanes used in the treatment of advanced breast and ovarian cancer. Health Technol Assess 4: 1-113, 2000.

2. Okamoto A, Nikaido T, Ochiai K, et al: Indoleamine 2,3dioxygenase serves as a marker of poor prognosis in gene expression profiles of serous ovarian cancer cells. Clin Cancer Res 11: 6030-6039, 2005.
3. Uyttenhove C, Pilotte L, Theate I, et al: Evidence for a tumoral immune resistance mechanism based on tryptophan degradation by indoleamine 2,3-dioxygenase. Nat Med 9: 1269-1274, 2003.

4. Muller AJ, DuHadaway JB, Donover PS, Sutanto-Ward E and Prendergast GC: Inhibition of indoleamine 2,3-dioxygenase, an immunoregulatory target of the cancer suppression gene Bin1, potentiates cancer chemotherapy. Nat Med 11: 312-319, 2005.

5. Ono K, Tanaka T, Tsunoda T, et al: Identification by cDNA microarray of genes involved in ovarian carcinogenesis. Cancer Res 60: 5007-5011, 2000

6. Enomoto T, Weghorst CM, Inoue M, Tanizawa O and Rice JM: $\mathrm{K}$-ras activation occurs frequently in mucinous adenocarcinomas and rarely in other common epithelial tumors of the human ovary. Am J Pathol 139: 777-785, 1991.

7. Fujita M, Enomoto T, Inoue M, et al: Alteration of the p53 tumor suppressor gene occurs independently of K-ras activation and more frequently in serous adenocarcinomas than in other common epithelial tumors of the human ovary. Jpn J Cancer Res 85: 1247-1256, 1994

8. Ichikawa Y, Nishida M, Suzuki H, et al: Mutation of K-ras protooncogene is associated with histological subtypes in human mucinous ovarian tumors. Cancer Res 54: 33-35, 1994.

9. Pieretti M, Cavalieri C, Conway PS, Gallion HH, Powell DE and Turker MS: Genetic alterations distinguish different types of ovarian tumors. Int J Cancer 64: 434-440, 1995.

10. Teneriello MG, Ebina M, Linnoila RI, et al: p53 and Ki-ras gene mutations in epithelial ovarian neoplasms. Cancer Res 53: 3103-3108, 1993.

11. Dent J, Hall GD, Wilkinson N, et al: Cytogenetic alterations in ovarian clear cell carcinoma detected by comparative genomic hybridisation. Br J Cancer 88: 1578-1583, 2003.

12. Enomoto T, Kuragaki C, Yamasaki M, et al: Is clear cell carcinoma and mucinous carcinoma of the ovary sensitive to combination chemotherapy with paclitaxel and carboplatin? Proc Am Soc Clin Oncol 22: 447, 2003.

13. Clark TG, Stewart ME, Altman DG, Gabra H and Smyth JF: A prognostic model for ovarian cancer. Br J Cancer 85: 944-952, 2001.

14. Sugiyama T, Kamura T, Kigawa J, et al: Clinical characteristics of clear cell carcinoma of the ovary: a distinct histologic type with poor prognosis and resistance to platinum-based chemotherapy. Cancer 88: 2584-2589, 2000.

15. Goff BA, Sainz de la Cuesta R, Muntz HG, et al: Clear cell carcinoma of the ovary: a distinct histologic type with poor prognosis and resistance to platinum-based chemotherapy in stage III disease. Gynecol Oncol 60: 412-417, 1996.

16. Schwartz DR, Kardia SL, Shedden KA, et al: Gene expression in ovarian cancer reflects both morphology and biological behavior, distinguishing clear cell from other poor-prognosis ovarian carcinomas. Cancer Res 62: 4722-4729, 2002.

17. Tsuda H, Ito YM, Ohashi Y, et al: Identification of overexpression and amplification of ABCF2 in clear cell ovarian adenocarcinomas by cDNA microarray analyses. Clin Cancer Res 11: 6880-6888, 2005

18. Stern RC, Dash R, Bentley RC, Snyder MJ, Haney AF and Robboy SJ: Malignancy in endometriosis: frequency and comparison of ovarian and extraovarian types. Int J Gynecol Pathol 20: 133-139, 2001.

19. Sekizawa A, Amemiya S, Otsuka J, et al: Malignant transformation of endometriosis: application of laser microdissection for analysis of genetic alterations according to pathological changes. Med Electron Microsc 37: 97-100, 2004.

20. Kato N, Sasou S and Motoyama T: Expression of hepatocyte nuclear factor-1beta (HNF-1beta) in clear cell tumors and endometriosis of the ovary. Mod Pathol 19: 83-89, 2006.

21. Munn DH, Sharma MD, Lee JR, et al: Potential regulatory function of human dendritic cells expressing indoleamine 2,3dioxygenase. Science 297: 1867-1870, 2002.

22. Friberg M, Jennings R, Alsarraj M, et al: Indoleamine 2,3dioxygenase contributes to tumor cell evasion of $\mathrm{T}$ cellmediated rejection. Int J Cancer 101: 151-155, 2002.

23. Munn DH, Sharma MD, Hou D, et al: Expression of indoleamine 2,3-dioxygenase by plasmacytoid dendritic cells in tumor-draining lymph nodes. J Clin Invest 114: 280-290, 2004.

24. Bai F, Feng J, Cheng Y, Shi J, Yang R and Cui H: Analysis of gene expression patterns of ovarian cancer cell lines with different metastatic potentials. Int J Gynecol Cancer 16: 202-209, 2006. 
25. Lancaster JM, Dressman HK, Clarke JP, et al: Identification of genes associated with ovarian cancer metastasis using microarray expression analysis. Int J Gynecol Cancer 16: 1733-1745, 2006.

26. Takei Y, Mizukami H, Saga Y, et al: Overexpression of a hybrid gene consisting of the amino-terminal fragment of urokinase and carboxyl-terminal domain of bikunin suppresses invasion and migration of human ovarian cancer cells in vitro. Int J Cancer 113: 54-58, 2005

27. Luo LY, Katsaros D, Scorilas A, et al: The serum concentration of human kallikrein 10 represents a novel biomarker for ovarian cancer diagnosis and prognosis. Cancer Res 63: 807-811, 2003.
28. Luo LY, Katsaros D, Scorilas A, et al: Prognostic value of human kallikrein 10 expression in epithelial ovarian carcinoma. Clin Cancer Res 7: 2372-2379, 2001

29. Takano M, Fujii K, Kita T, Kikuchi Y and Uchida K: Amplicon profiling reveals cytoplasmic overexpression of MUC1 protein as an indicator of resistance to platinum-based chemotherapy in patients with ovarian cancer. Oncol Rep 12: 1177-1182, 2004.

30. Matsuzaki H, Kobayashi H, Yagyu T, et al: Plasma bikunin as a favorable prognostic factor in ovarian cancer. J Clin Oncol 23: 1463-1472, 2005. 